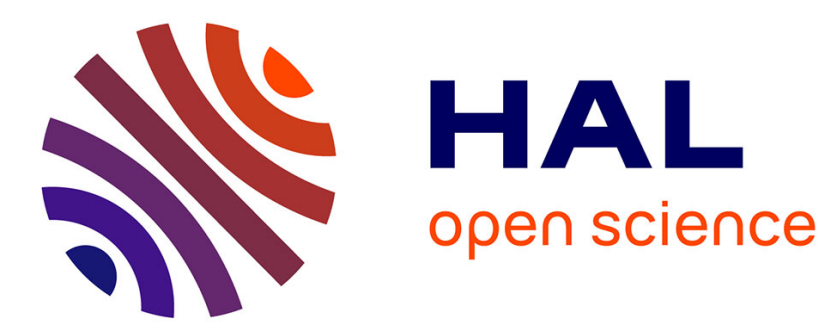

\title{
Coherent light transmission by a dew pattern
}

\author{
Vadim Nikolayev, P. Sibille, Daniel Beysens
}

\section{To cite this version:}

Vadim Nikolayev, P. Sibille, Daniel Beysens. Coherent light transmission by a dew pattern. Optics Communications, 1998, 10.1016/S0030-4018(98)00051-0 . hal-01261262

\section{HAL Id: hal-01261262 \\ https://hal.science/hal-01261262}

Submitted on 28 Jan 2016

HAL is a multi-disciplinary open access archive for the deposit and dissemination of scientific research documents, whether they are published or not. The documents may come from teaching and research institutions in France or abroad, or from public or private research centers.
L'archive ouverte pluridisciplinaire HAL, est destinée au dépôt et à la diffusion de documents scientifiques de niveau recherche, publiés ou non, émanant des établissements d'enseignement et de recherche français ou étrangers, des laboratoires publics ou privés.

\section{(이) $\$$}

Distributed under a Creative Commons Attribution - NonCommercial - NoDerivatives| 4.0 


\title{
Coherent light transmission by a dew pattern
}

\author{
Vadim S. Nikolayev \\ Present address: Service de Physique de l'Etat Condensé, CEA, CNRS, Université Paris-Saclay, CEA Saclay, 91191 Gif-sur-Yvette Cedex, France \\ Département de Recherche Fondamentale sur la Matière Condensée, CEA Grenoble, 38054, Grenoble Cedex 9, France
}

Patrick Sibille

Service de Physique de l'Etat Condensé, CEA Saclay, 91191 Gif-sur-Yvette Cedex, France

Daniel A. Beysens

Département de Recherche Fondamentale sur la Matière Condensée, CEA Grenoble, 38054, Grenoble Cedex 9, France

\begin{abstract}
We study both theoretically and experimentally the transmission of coherent light by a drop pattern (dew). The theory is based on the Kirchhoff scalar approach to diffraction. The polarization of the diffracted wave in the zero diffraction order is analyzed separately. The intensity in the zero diffraction order in the far zone is an oscillatory function of the drop size. These oscillations are observed with a pattern of water drops growing on glass. The model allows the evolution of the important parameters of the drop pattern (average radius and surface coverage) to be obtained from the light intensity in the zero diffraction order.
\end{abstract}

\section{Introduction}

Depending on the wettability of the surface on which vapor condenses, dew forms a transparent film or a diffuse assembly of droplets. The knowledge of the properties of dew, "breath figures" or, generally speaking, dropwise condensation, opens a vast field of applications ranging from high-technology processes of film growth [1] to soil desinfection in agronomy [2], sterilization in pharmacology [3] and water recovery in dew condensers [4]. The optical properties of dew have received considerable attention from the scientists who studied the natural physical effects (see, e.g. [5, 6]) and from those who are interested in industrial applications [7, 8].

The morphology and kinetics of dew formation were investigated extensively both theoretically and experimentally (see e.g. $[9,10]$ and refs. therein). Its growth can be characterized by several physical values, the most important of which are the mean radius $\langle a\rangle$ of the drops and the surface coverage $\varepsilon^{2}$, which is the fraction of surface area covered by the projections of the drops on the surface.

Two regimes of growth can be identified. At the beginning of the condensation process the drops grow independently, $\langle a\rangle$ follows a power law $\langle a\rangle \sim t^{\mu_{0}}$, where $t$ is the elapsed time, and the surface coverage increases. When the temperature of the substrate is kept constant, $\mu_{0}=\frac{1}{3}$. When the drop radius becomes large enough, coalescences between drops occur and the exponent changes to another value $\mu=3 \mu_{0}$. The growth is then self-similar and the surface coverage reaches a saturation value

Email address: vadim.nikolayev@cea.fr (Vadim S. Nikolayev) $\varepsilon_{\infty}^{2}$ as well as all the statistical characteristics of the drop pattern, except $\langle a\rangle$. When the surface is ideally smooth and clean $\varepsilon_{\infty}^{2} \approx 0.55$ independently of the wetting properties (characterized by the contact angle $\phi$, see Fig. 1). For a nonideal surface, the pinning of the contact lines by the surface heterogeneities leads to a hysteresis of the contact angle, i.e. to a significant difference between the advancing and receding contact angles. The shape of the drop is no more a spherical cap. In this case $\varepsilon_{\infty}^{2}$ becomes dependent on the hysteresis, and since the hysteresis effects are stronger for small contact angles [11], $\varepsilon_{\infty}^{2}$ becomes higher as $\phi$ is smaller. This dependence will be used in section 3 to determine the contact angle. In the analysis below, the drops are considered to be spherical caps and the influence of gravity, which is important only when the drop reaches a size of $\approx 2 \mathrm{~mm}$ (the water capillary length), is neglected.

\section{Theoretical description}

Despite its importance, the optical properties of a dew pattern has up to now been investigated to our knowledge by geometrical optics only $[2,6]$. Indeed, the light scattering by a random set of nearly hemispherical drops with different size is quite difficult to describe. Here we give a solution in a particular, but important, case: we calculate the intensity of the zero order of the far zone diffraction, i.e. the transmission of a coherent light through the dew pattern.

\subsection{Optical properties of a single drop}

Let a monochromatic linearly polarized plane wave with amplitude $\overrightarrow{E^{s}}$ travel through a transparent substrate and then fall 


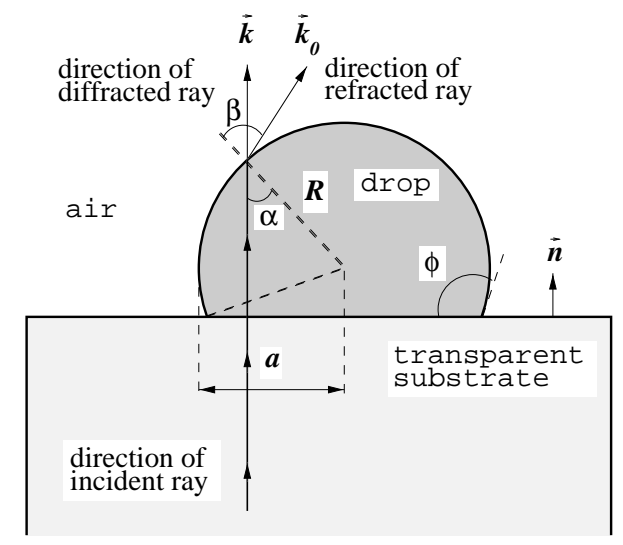

Figure 1: The geometry of a drop. See text for the explanations.

normally on the interface between the transparent substrate and a water drop (see Fig. 1). The wave is partially reflected, and the amplitude of the wave just after the plane substrate-water interface is [12] $2 n_{w} \vec{E}^{s} /\left(n_{w}+n_{s}\right)$, where $n_{s}$ and $n_{w}$ are the refractive indices of the substrate and water respectively. Passing through water, the wave gains a phase shift. Thus the complex amplitude of the wave just before the water-air interface is

$$
\vec{E}^{i}=2 n_{w} /\left(n_{w}+n_{s}\right) \vec{E}^{s} \exp \left(i k_{0} n_{w} l\right),
$$

where $k_{0}=2 \pi / \lambda, \lambda$ being the wavelength in vacuum, and

$$
l=\sqrt{R^{2}-r^{2}}-R \cos \phi,
$$

is the geometrical path of the ray inside the water drop. Here $r$ is the distance of the ray from the center of the drop (Fig. 1), $R$ is the radius of curvature of the drop and $\phi$ is the contact angle. Then the ray is refracted and its polarization changes according to the Fresnel formulae [12]. While the component of the field which lies in the plane of incidence is transformed according to

$$
E_{\|}^{t}=E_{\|}^{i} t_{\|} \quad \text { with } \quad t_{\|}=\frac{2 \sin \beta \cos \alpha}{\sin (\alpha+\beta) \cos (\alpha-\beta)},
$$

the component which lies in the perpendicular plane is

$$
E_{\perp}^{t}=E_{\perp}^{i} t_{\perp}, \quad \text { with } \quad t_{\perp}=\frac{2 \sin \beta \cos \alpha}{\sin (\alpha+\beta)},
$$

where $\alpha$ and $\beta$ are the angles of incidence and refraction respectively (see Fig. 1). These angles are related through the Descartes-Snellius law

$$
n_{w} \sin \alpha=n_{a} \sin \beta,
$$

where $n_{a}$ is the refractive index of air and $\alpha$ is defined directly by $r$ :

$$
\sin \alpha=r / R \text {. }
$$

We consider a polar coordinate system in a plane parallel to the substrate, see Fig. 2. It is chosen in such a way that its reference point coincides with the drop center $O$ and the reference direction (called the $\xi$-axis below) is the direction of polarization of the incident wave. Then for an arbitrary point with coordinates $(r, \psi)$

$$
E_{\|}^{i}=E^{i} \cos \psi, \quad E_{\perp}^{i}=E^{i} \sin \psi
$$

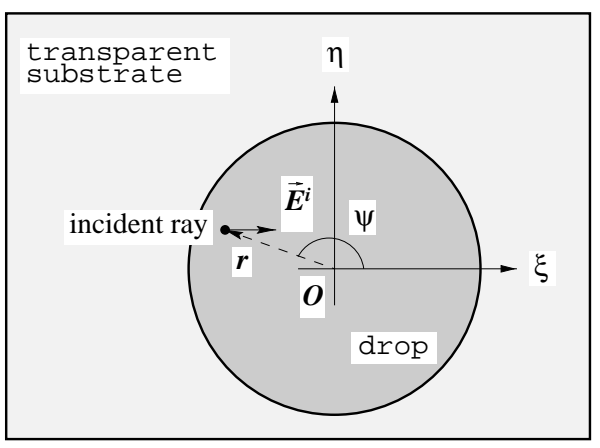

Figure 2: The top view of the drop in Fig. 1 with $\xi O \eta$ Cartesian and $r O \psi$ polar coordinate systems.

Using the $\xi O \eta$ Cartesian coordinate system (Fig. 2), it is easy to check with the help of Eqs. 3, 4 and 7 that

$$
\begin{gathered}
E_{\xi}^{t}=E^{i}\left(t_{\|} \cos ^{2} \psi+t_{\perp} \sin ^{2} \psi\right), \\
E_{\eta}^{t}=E^{i}\left(t_{\perp}-t_{\|}\right) \sin \psi \cos \psi .
\end{gathered}
$$

Waves of different polarization do not interfere and can be analyzed separately. The $\eta$-components of the waves, emitted by two mirror symmetrical points $(r, \psi)$ and $(r,-\psi)$ of the drop surface satisfy the following relation

$$
E_{\eta}^{t}(r, \psi)+E_{\eta}^{t}(r,-\psi)=0 .
$$

Because the drop is a spherical cap and the incidence is normal, the angles $\alpha$ and $\beta$ as defined by Eqs. 5 and 6 are independent of the $\psi$-coordinate of the incident ray (Fig. 2). Thus $t_{\|}$ and $t_{\perp}$ as defined in (3) and (4) are independent of $\psi$ too. Then (10) follows from (9). Therefore, as far as only the zero order of diffraction is concerned, the $\eta$-polarized components of the waves which come from two these points annihilate each other. Since this argument is applicable to every two symmetrical points, the $\eta$-polarized components of the waves yield no contribution. Therefore, the wave in the zero order of diffraction (unlike any other point in the image plane) is polarized in the $\xi$-direction, i.e. in the direction of polarization of the incident wave. This allows the index $\xi$ to be omitted hereafter and the scalar wave theory for the $\xi$-component to be applied. The key parameter of this theory [12] is the complex transparency of the drop which we introduce now.

The visible radius of the drop $a$ can be related to its curvature radius $R$ through the contact angle $\phi$ (see Fig. 1):

$$
\zeta \equiv \frac{a}{R}= \begin{cases}\sin \phi, & \text { if } \phi<\pi / 2 \\ 1, & \text { otherwise }\end{cases}
$$

We neglect the contribution of the rays which are multiply reflected or refracted before leaving the drop. Each reflection or refraction decreases strongly the amplitude of the corresponding fields according to the Fresnel formulae, the angles of reflection or refraction being there large. An additional attenuation is due to the light absorption by water. Another reason for amplitude decrease is the angle factor $K$, which is small for the large angles of refraction (see below). 
When the angle of incidence at the surface of the drop $\alpha$ (see Fig. 1) is larger than $\alpha_{c r}$, which is defined by the relation

$$
\sin \alpha_{c r}=\gamma \equiv n_{a} / n_{w},
$$

full internal reflection occurs. Therefore, two regions can be defined in a drop: an inner transparent region of radius $a^{\prime}<a$ and an outer annular "black" region. The ratio $\chi=a^{\prime} / R$ is easy to determine by using Fig. 1 after straightforward geometrical arguments:

$$
\chi= \begin{cases}\gamma, & \text { if } \alpha_{c r}<\phi<\pi-\alpha_{c r}, \\ \sin \phi, & \text { otherwise. }\end{cases}
$$

The contribution of the rays which have to cross an air layer before entering the drop when $\phi>\pi / 2$ is neglected in accordance with our "single-refraction" assumption.

The complex transparency of the drop with visible radius $a$ can be written as

$$
\tau_{a}(\vec{r})= \begin{cases}\frac{n_{w}\left(n_{a}+n_{s}\right)}{n_{a}\left(n_{w}+n_{s}\right)} C(\psi) \mathrm{e}^{i k_{0}\left(n_{w}-n_{a}\right) l-\mu l} & \text { if } 0 \leq r \leq a^{\prime}, \\ 0, & \text { if } a^{\prime}<r<a, \\ 1, & \text { if } r \geq a,\end{cases}
$$

where $\mu$ is the coefficient of light absorption. It is negligible for visible light and we put $\mu=0$ in the following. The function $C(\psi)$ is given by

$$
C(\psi)=t_{\|} \cos ^{2} \psi+t_{\perp} \sin ^{2} \psi .
$$

Eq. 13 follows from (8) after substitution of (1) and division by the amplitude of the wave unperturbed by the presence of the drop.

\subsection{Diffraction by a single drop.}

Kirchhoff's formula [12], reduced for the diffraction in the far zone, gives the following expression for the amplitude depending on the direction to the point of observation $\vec{k}$ :

$$
E(\vec{k})=\iint \tau_{a}(\vec{r}) K \exp \left[-i n_{a} \vec{r} \cdot\left(\vec{k}_{0}+\vec{k}\right)\right] \mathrm{d} \vec{r},
$$

where $\tau_{a}$ is the complex transparency of the object and $K$ is the angle factor given by

$$
K=\frac{1}{2 k_{0}}\left(\vec{k}_{0}+\vec{k}\right) \cdot \vec{n} .
$$

The integration in (15) is performed over the reference plane parallel to the substrate, $\vec{n}$ is a unit normal to it (Fig. 1), $\vec{k}_{0}$ is a normal vector to the wave front which falls on the reference plane, $\left|\vec{k}_{0}\right|=|\vec{k}|=k_{0}$. Expression (15) holds just for the small scattering angles, i.e. for $\vec{k}$ close to $k_{0} \vec{n}$ (in the case considered here $\vec{k}=k_{0} \vec{n}$ exactly). Thus (16) reads

$$
K=\frac{1}{2}\left(\frac{\vec{k}_{0}}{k_{0}} \cdot \vec{n}+1\right) .
$$

For the diffraction by the drop (see Fig. 1) this expression reduces to

$$
K=\frac{1}{2}[\cos (\beta-\alpha)+1]
$$

In the familiar case of the Fraunhofer diffraction by a hole or by a quasi-flat phase object, $\vec{k}_{0}$ does not depend on $\vec{r}, \vec{k}_{0}=k_{0} \vec{n}$, and $E(\vec{k})$ is just the Fourier-transform of $\tau_{a}(\vec{r})$. In the case of a drop, the direction of $\vec{k}_{0}$ is a function of $\vec{r}$, and $E(\vec{k})$ is the Fourier transform of the function

$$
\tilde{\tau}_{a}(\vec{r})=\tau_{a}(\vec{r}) K \exp \left(-i n_{a} \vec{r} \cdot \vec{k}_{0}\right)
$$

Note that $\tilde{\tau}_{a}(\vec{r})=\tau_{a}(\vec{r})=1$ outside the drop.

\subsection{Zero-order diffraction by a dew pattern}

The theory of diffraction by an assembly of objects is presented in [13] under the assumption of applicability of the conditions of Fraunhofer diffraction when the amplitude in the image plane is the Fourier transform of the object transparency. It is shown in [13] that the intensity in the zero diffraction order is defined by the so-called "Debye volume scattering term" which can be written within a constant as

$$
I_{0} \equiv|E(\vec{k}=0)|^{2}=|\langle\tau\rangle|^{2},
$$

where

$$
\langle\tau\rangle=\frac{1}{s_{1}}\left\langle\int_{\left(s_{1}\right)} \tau_{a}(\vec{r}) \mathrm{d} \vec{r}\right\rangle .
$$

Here $s_{1}$ is the total illuminated area of the substrate divided by the total number of the drops and the angle brackets mean an average over the size distribution. Although the theory was developed for a real transparency $\tau_{a}(\vec{r})$ (called "density" in [13]), it can be easily generalized for the complex case. The invariance with respect to the spatial distribution of the drops is an interesting feature of expression (19).

As already discussed, the function $\tilde{\tau}_{a}(\vec{r})$ should be used in (20) instead of $\tau_{a}(\vec{r})$. It is easy to check that this function is radially symmetric which is a necessary condition of the applicability of the results [13]. Thus

$$
\begin{aligned}
\tau=1 & -\frac{\pi a^{2}}{s_{1}}+\frac{1}{s_{1}} \frac{n_{a}+n_{s}}{n_{w}+n_{s}} \frac{n_{w}}{n_{a}} \\
& \int_{0}^{a^{\prime}} J K r \exp \left\{i k_{0}\left[\left(n_{w}-n_{a}\right) l+n_{a} r \sin (\beta-\alpha)\right]\right\} \mathrm{d} r,
\end{aligned}
$$

where

$$
J=\int_{0}^{2 \pi} C(\psi) \mathrm{d} \psi=\pi\left(t_{\|}+t_{\perp}\right)
$$

In order to find an average of (21), one needs to introduce the distribution $H(a)$, which defines the probability to find a drop with the visible radius $a$. Since the two important physical values are the average size $\langle a\rangle$ and the polydispersity $g=$ $\left(\left\langle a^{2}\right\rangle-\langle a\rangle^{2}\right)^{1 / 2} /\langle a\rangle$, a distribution with only two free parameters can be considered. We choose for this purpose the Maxwellian distribution as described in [13]:

$$
H(a)=\frac{B(m)}{a_{0}}\left(\frac{a}{a_{0}}\right)^{m} \mathrm{e}^{-\left(a / a_{0}\right)^{2}},
$$


where $B(m)$ is a normalization constant. The free parameters $m \geq-1$ and $a_{0}$ can be related to $g$ and $\langle a\rangle$ [13] through

$$
\begin{array}{r}
g \approx(2 m+2)^{-1 / 2}, \\
\langle a\rangle \approx a_{0}[(m+0.5) / 2]^{1 / 2} .
\end{array}
$$

These approximations become accurate within less than $1 \%$ when $m>4$.

The mean surface coverage $\varepsilon^{2}$ is related to $s_{1}$ by $\varepsilon^{2}=\pi\left\langle a^{2}\right\rangle / s_{1}$. Note that it is defined here by the projected area covered by the drops, not by the actual area in contact with water. The set of Eqs. 21-24 allows one to calculate $\langle\tau\rangle$ as a function of the parameters $\langle a\rangle, g$ (or $\left.a_{0}, m\right)$ and $\varepsilon^{2}$ :

$$
\begin{gathered}
\langle\tau\rangle=1-\varepsilon^{2}+\frac{\varepsilon^{2}}{\zeta^{2} \gamma^{2}} \frac{n_{a}+n_{s}}{n_{w}+n_{s}} B(m+2) \\
\int_{\sqrt{1-\chi^{2}}}^{1} \mathrm{~d} x \frac{x^{2}\left(1+\gamma+v x-x^{2}\right)^{2}}{(v+x)\left(v x+1-x^{2}\right)} \int_{0}^{\infty} \mathrm{d} y y^{m+2} \\
\exp \left\{i y n_{w} \frac{2 \pi a_{0}}{\zeta \lambda}\left[(1-\gamma)(x-\cos \phi)-\left(1-x^{2}\right)(x-v)\right]-y^{2}\right\},
\end{gathered}
$$

where $v=\sqrt{x^{2}+\gamma^{2}-1}$. The intensity of the zero diffraction order $I_{0}$ can now be calculated by using (19).

Let us analyze now the function $I_{0}(\langle a\rangle)$ with $g$ and $\varepsilon^{2}$ as parameters. This choice reflects the growth kinetics of the selfsimilar regime (see section 1$)$ when the only growing parameter is $\langle a\rangle$. Oscillations of the function $I_{0}(\langle a\rangle)$ can occur due to the exponential dependence of $i a_{0}$, the latter being related to $\langle a\rangle$ by (24).

The asymptotic values of the function $I_{0}(\langle a\rangle)$ can be calculated explicitly:

$$
\begin{array}{r}
I_{0}(\langle a\rangle \rightarrow 0)=\left[1-\varepsilon^{2}+\frac{\varepsilon^{2}}{\zeta^{2} \gamma^{2}} \frac{n_{a}+n_{s}}{n_{w}+n_{s}}\right. \\
\left.\int_{\sqrt{1-\chi^{2}}}^{1} \frac{x^{2}\left(1+\gamma+v x-x^{2}\right)^{2}}{(v+x)\left(v x+1-x^{2}\right)} \mathrm{d} x\right]^{2}, \\
I_{0}(\langle a\rangle \rightarrow \infty)=\left(1-\varepsilon^{2}\right)^{2} .
\end{array}
$$

The interesting feature of these expressions is that they do not depend on the polydispersity and, therefore, on the form of the function $H(a)$. While (26) is not particularly useful for practical purposes (since $\varepsilon^{2}=0$ always when $\langle a\rangle=0$, giving $\left.I_{0}=1\right)$, Eq. 27 is more interesting because it relates $I_{0}(t \rightarrow \infty)$ to $\varepsilon_{\infty}^{2}$. It is easy to see that the value (27) gives the geometrical optics limit and corresponds to the transparency of an assembly of black spots. In this case $\langle\tau\rangle=1-\varepsilon^{2}$ and $I_{0}$ depends either on the shape or on the size of the spots through $\varepsilon^{2}$ only. One can therefore conclude that the presence of the oscillations is connected to the phase shift of the transmitted wave by the drops.

The results of the calculations of the function $I_{0}(\langle a\rangle)$ for different constant values of the other parameters are presented

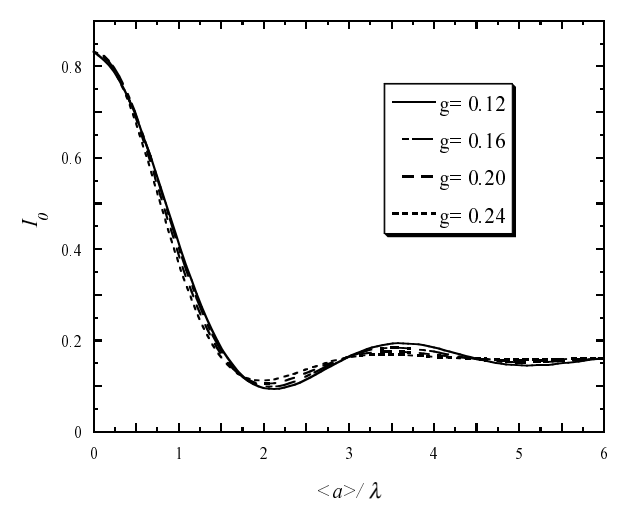

Figure 3: The function $I_{0}(\langle a\rangle)$ for $\varepsilon^{2}=0.6, \phi=90^{\circ}$ and for different values of $g$.

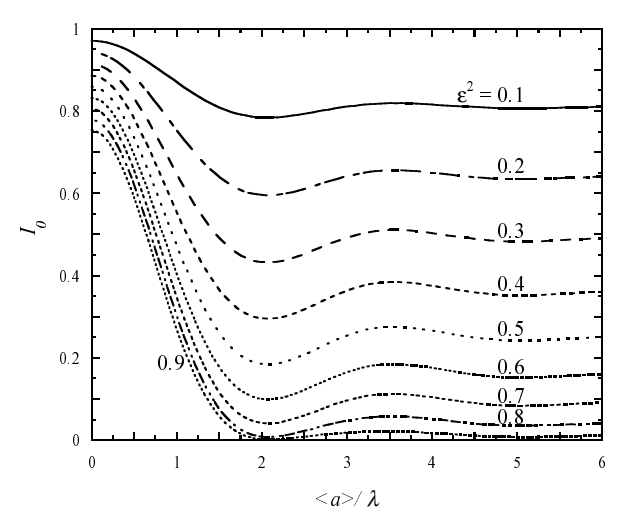

Figure 4: The function $I_{0}(\langle a\rangle)$ for $g=0.16, \phi=90^{\circ}$ and for different values of $\varepsilon^{2}$ 


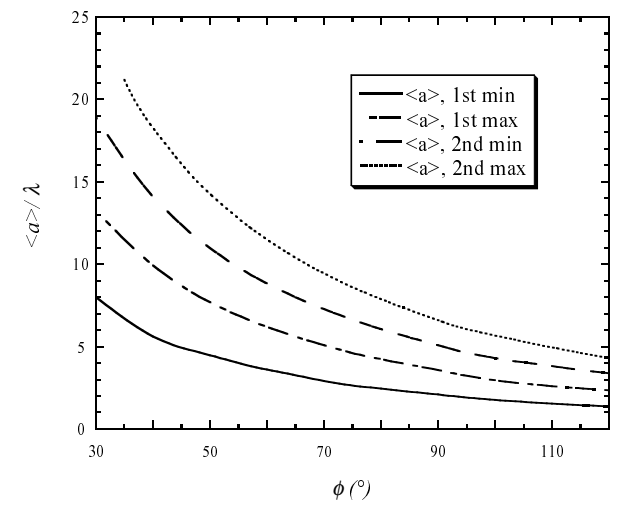

Figure 5: The positions of the four first extrema of the function $I_{0}(\langle a\rangle)$ versus the contact angle $\phi$ for $g=0.16$ and $\varepsilon^{2}=0.6$.

in Figs. 3 and 4. We recall here that in a real condensation experiment $g$ and $\varepsilon^{2}$ are not constant in the beginning of the growth (see section 1). Thus the curves presented in Figs. 3 and 4 cannot be observed experimentally. For example, it is generally impossible to obtain $\varepsilon^{2}=0.6$ for small values of $\langle a\rangle$, while the curve which corresponds to this surface coverage is plotted for all values of $\langle a\rangle$ in Fig. 4.

Figs. 3 and 4 show that the amplitude of the oscillations of the function $I_{0}(\langle a\rangle)$ is controlled mainly by the polydispersity, while the "mean" level, around which the intensity oscillates, depends strongly on $\varepsilon^{2}$. A large polydispersity suppresses the oscillations. The positions of the extrema of $I_{0}(\langle a\rangle)$ are practically independent of $\varepsilon^{2}$ and $g$ over a wide range of the parameters. However, the dependence of the positions of the extrema on the contact angle $\phi$ is stronger. It is presented in Fig. 5. These features allow the values of the parameters $\langle a\rangle$ and $\varepsilon^{2}$ to be determined experimentally as will be demonstrated below.

\section{Experimental: determination of the characteristics of a dew pattern}

The sketch of the experimental setup is shown in Fig. 6. It is derived from the setup in [14]. It permits simultaneous observation of the scattered light and measurement of the intensity on the optical axis. No direct visualization of the droplets was sought. Dew is produced by blowing air saturated with water vapor on a cooled glass slide coated with a hydrophobic layer of silane, which plays the role of the substrate from the optical point of view. The process of glass coating is described in [10]. The coherent light beam generated by a $5 \mathrm{~mW} \mathrm{He}-\mathrm{Ne}$ laser $(\lambda=0.6328 \mu \mathrm{m})$ passes through the glass plate and the dew pattern. The diffracted light is collected by a large lens $(10 \mathrm{~cm}$ diameter) placed at its focal length. The diffraction pattern is visualized through a translucid screen. The pictures are recorded by a CCD video camera and digitized for further analysis. A mirror placed at the center of the lens deviates the transmitted light perpendicularly towards a photodiode. The distance between the photodiode and the mirror is chosen so as to provide the conditions of Fraunhofer diffraction [12] in the photodiode plane. The size of the mirror and the pin-hole in front of the

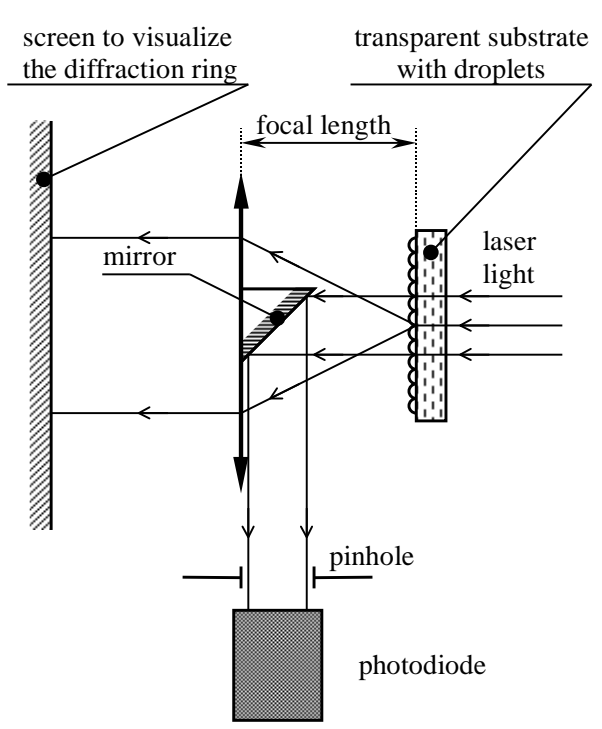

Figure 6: Schematic diagram of the experimental setup.

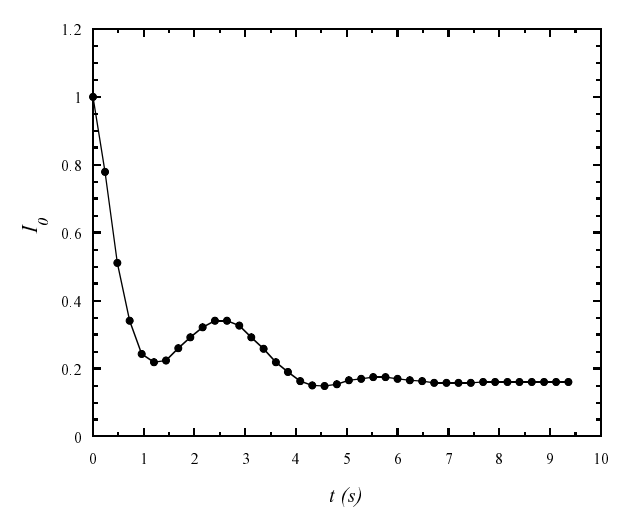

Figure 7: Experimental time evolution of $I_{0}$. The data are normalized by the value $I_{0}$ at $t=0$.

photodiode correspond to the width of the laser beam. This allows all the light to be collected in the absence of scattering by dew. When condensation starts, the photodiode measures the intensity of the zero diffraction order. The temperature of the glass slide is stabilized by an attached large copper block connected to the Peltier element and a power supply. The temperature is controlled during the experiment. The video image and the photodiode signal are simultaneously recorded when vapor is blown onto the glass slide.

A typical $I_{0}$ recording is shown in Fig. 7. The time evolution of $I_{0}$ can be understood on the basis of the model considered in the previous section. The sharp decrease of the "mean" value with respect to the oscillations reflects the evolution of the surface coverage $\varepsilon^{2}$. The latter increases until it reaches the value $\varepsilon_{\infty}^{2}$. Several oscillations appear, in agreement with the above theoretical prediction. Moreover, the model gives an opportunity to determine the parameters of the dew pattern by using the time dependence of $I_{0}$ (Fig. 7). The algorithm for the determination of $\varepsilon^{2}$ and $\langle a\rangle$ as functions of time $t$ can be described as follows.

1. The value of $I_{0}$ at large time corresponds to the saturation 


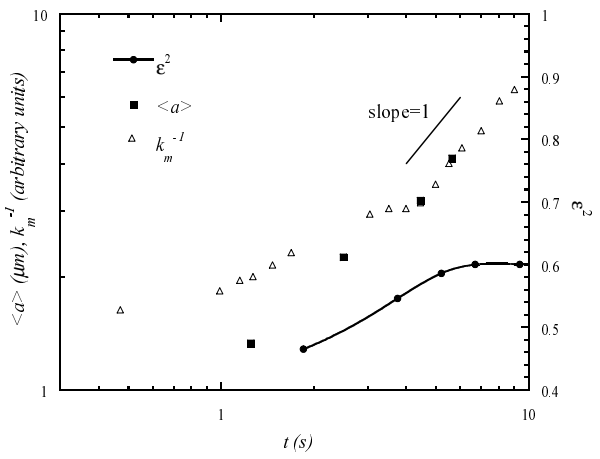

Figure 8: Time evolution of the wave vector modulus $k_{m}$, the drop surface coverage $\varepsilon^{2}$ and the mean radius of the droplets $\langle a\rangle$.

value (27). Here we obtain $I_{0}=0.16$, thus $\varepsilon_{\infty}^{2}=0.6$.

2 . The data [11] where $\varepsilon_{\infty}^{2}$ is plotted versus contact angle shows that this value corresponds to an average contact angle $\phi \simeq 90^{\circ}$. We thus use the data calculated for this particular value of $\phi$.

3. Because the positions of the extrema of the function $I_{0}(\langle a\rangle)$ are nearly independent of $\varepsilon^{2}$ and $g$, we can determine with high accuracy the values of $\langle a\rangle$ at the times which correspond to the extrema of the function $I_{0}(t)$. They are plotted versus $t$ in Fig. 8 .

Fig. 3 shows that the value of $I_{0}$ is independent of the polydispersity $g$ in the inflection points [15] of the function $I_{0}(\langle a\rangle)$. By making use of this property, we can obtain the values of $\varepsilon^{2}$ by the following steps.

4. The time values at the inflection points and the corresponding values of the intensity are extracted from Fig. 7.

5. The values of $\langle a\rangle$ at these values of time are determined by the interpolation of the dependence $\langle a\rangle(t)$ obtained in step 3 .

6. Eq. 25 can be rewritten in the form

$$
\langle\tau\rangle=1-\varepsilon^{2}+\varepsilon^{2}(A+i D),
$$

where the quantities $A$ and $D$ are independent of $\varepsilon^{2}$. Then (19) with the substitution of (28) can be easily solved for $\varepsilon^{2}$ :

$$
\varepsilon^{2}=\left\{1-A-\left\{I_{0}\left[D^{2}+(1-A)^{2}\right]-D^{2}\right\}^{1 / 2}\right\} / 2 /\left[D^{2}+(1-A)^{2}\right] .
$$

Since $A$ and $D$ can be calculated for an arbitrary value of $g$ (we took $g=0.16$ ) and values of $\langle a\rangle$, obtained in the step 5, the corresponding values of $\varepsilon^{2}$ can be determined from (29) by using the values of $I_{0}$ from step 4 . These data for $\varepsilon^{2}(t)$ are plotted in Fig. 8 with the saturation value from the step 1 .

One can see that the curve $\varepsilon^{2}(t)$ is similar to the curve obtained by numerical simulation [10]. The time evolution of $\langle a\rangle$ can be compared with that of $k_{m}^{-1}$. The value of $k_{m}$ is defined as the wavevector corresponding to the maximum intensity in the diffraction pattern (ring). It is obtained by the image analysis of the diffraction picture as recorded by the video camera. One can see that for large times the growth laws of $k_{m}^{-1}$ and $\langle a\rangle$ are the same. They correspond to the growth exponent $\mu \simeq 1$.

\section{Conclusions}

The theoretical model developed here deals with the intensity of the coherent light transmitted by a time dependent dew pattern. The theory explains the oscillations of the intensity observed in the zero diffraction order in the far zone. In addition, the model allows the evolution of the two most important parameters of the drop pattern to be assessed: the average drop radius and the drop surface coverage.

V. N. would like to thank the direction of DRFMC, CEA/Grenoble for its kind hospitality and the Ministère de l'Enseignement Supérieur et de la Recherche of France for financially supporting this work.

[1] D. Beysens, C. M. Knobler and H. Schaffar, Phys. Rev. B 41 (1990) 9814.

[2] A. Jaffrin, S. Makhlouf, C. Scotto La Massese, A. Bettachini et R. Voisin, Agronomie 9 (1989) 729.

[3] M. Marcos-Martin, A. Bardat, R. Schmitthaeusler and D. Beysens, Pharm. Techn. Eur. 8 (1996) 24.

[4] V. S. Nikolayev, D. Beysens, A. Gioda, I. Milimouk, E. Katiushin and J. P. Morel, Journal of Hydrology 182 (1996) 19.

[5] A. B. Fraser, Applied Optics 33 (1994) 4539.

[6] B. J. Briscoe and K. P. Galvin, Solar Energy 46 (1991) 191.

[7] J. G. Pieters, J. M. Deltour and M. J. Debruyckere, J. Phys. III (France) 6 (1996) 975.

[8] S. Matsumoto and S. Toyooka, Opt. Commun. 91 (1992) 5.

[9] B. J. Briscoe and K. P. Galvin, Phys. Rev. A 43 (1991) 1906.

[10] D. Fritter, C. M. Knobler and D. A. Beysens, Phys. Rev. A 43 (1991) 2858.

[11] H. Zhao and D. Beysens, Langmuir 11 (1995) 627.

[12] M. Born and E. Wolf, Principles of Optics (Pergamon Press, Oxford, 1980).

[13] R. Hosemann and S. N. Bagchi, Direct Analysis of Diffraction by Matter (North-Holland, Amsterdam, 1962).

[14] F. Perrot and D. Beysens, Rev. Sci. Instrum. 58 (1987) 183.

[15] The only exception is the first inflection point, not used in the following. 